\title{
Could Antimullerian Hormone Play a Role in Fertilization Failure in ICSI Application
}

\author{
Tulay Irez ${ }^{1, ~ *, ~ O n u r ~ G u ̈ r a l p ~}{ }^{2}$, Hakan Yelke ${ }^{3}$, Semra Kahraman $^{4}$ \\ ${ }^{1}$ Department of Histology and Embryology, Medical Faculty, Istanbul Yeni Yuzyil University, Istanbul, Turkey \\ ${ }^{2}$ Department of Gynaecology \& Obstetrics, Medical Faculty, University of Oldenburg, Oldenburg, Germany \\ ${ }^{3}$ Department of IVF and Gynetics Center, Sisli Memorial Hospital, Istanbul, Turkey \\ ${ }^{4}$ Department of Gynecology and Obstetrics and IVF and Gynetics Center, Sisli Memorial Hospital, Istanbul, Turkey
}

\section{Email address:}

ireztulay@yahoo.com (T. Irez),dronur@hotmail.com (O. Güralp), hyelke@yahoo.com (H. Yelke), semkahraman@gmail.com (S. Kahraman) ${ }^{*}$ Corresponding author

\section{To cite this article:}

Tulay Irez, Onur Güralp, Hakan Yelke, Semra Kahraman. Could Antimullerian Hormone Play a Role in Fertilization Failure in ICSI Application. Advances in Bioscience and Bioengineering. Vol. 9, No. 3, 2021, pp. 87-91. doi: 10.11648/j.abb.20210903.14

Received: August 7, 2021; Accepted: August 20, 2021; Published: September 10, 2021

\begin{abstract}
Aim: Failed fertilization (FF) occurs in approximately 2-3\% of ICSI cycles and is mainly due to lack of oocyte activation. The reasons of total fertilization insufficiency in ICSI applications can be listed as mitosis errors, sperm aster formation defects, sperm decondensation defects, PN formation defects and oocyte activation defects. In this study, sperm functions, oocyte morphology, hormone levels and possible effects of gonadotropins used in the treatment were investigated retrospectively. Material and Methods: In this study, semen parameters and sperm functions, oocyte morphology, basal hormone values and treatment processes of 32 fertilization failure cases and 91 fertile controls were compared. Results: It was found that age of female and male, basal FSH value and gonadotropin used in the treatment process were higher in total fertilization failure cases compared to fertile controls $(p=0.004, p=0.041, p=0.008, p=0.004)$. Basal AMH level, total oocyte count, M2 oocyte count, quality oocyte count and normal ZP percentage were lower in fertilization failure cases $(\mathrm{p}=$ $0.002, p=0.000, p=0.000, p=0.008, p=0.000)$. There was no statistically significant difference between the two groups in terms of sperm functions. Conclusions: It was understood that high FSH and low AMH levels and high-dose gonadotropin treatment in cases with optimal sperm quality may cause oocyte cytoplasmic and zona pellucida abnormalities, and it should be discussed as a cause of fertilization failure.
\end{abstract}

Keywords: Antimullerian Hormone, Oocyte Abnormalities, Fertilization Failure

\section{Introduction}

With ICSI, the sperm is inserted directly into the oocyte cytoplasm and IVF failure is treated in this way. However, in some patients, insufficient fertilization is seen in all ICSItreated eggs. After ICSI oocyte activation is characterized by a two-stage increase pattern of intracellular $\mathrm{Ca}^{2+}$ concentrations. The first $\mathrm{Ca}^{2+}$ increase (triggers) originates from the oocyte cortex following sperm-oocyte membrane interaction and follows a series of shorter $\mathrm{Ca}^{2+}$ transient amplitudes (oscillators) that continue for 3-4 hours (oscillator) after 30 minutes [1]. Oscillator function is due to the release of a sperm-associated oocyte activation factor that requires oocyte to sustain recurrent $\mathrm{Ca}^{2+}$ releases from intracellular stores [2]. Inadequacies in this oocyte-related osillator function have been shown as a result of auxiliary oocyte activation studies [3].

Genetic parameters in many sperm-related studies in cases of fertilization insufficiency and Torra-Massana M. et al. (2019) have shown that phospholipase $\mathrm{C}$ zeta 1 mutations are associated with insufficiency [4]. Fertilization deficiency is frequently observed in globozoospermic cases and studies have reported a multigenic defect due to acrosomal insufficiency in globozoospermic cases [5]. Therefore, Therefore, it is aimed to perform a retrospective study in young women with FF and patients with semen parameters within the acceptable range.

The role of AMH in influencing oocyte quality and oocyte 
activation mechanism in fertilization is unknown. Recently Zhang et al. suggested that AMH improved oocyte quality by upregulating GDF9 and BMP15 expressions in oocyte in vitro maturation studies [6]. Furthermore, it is found that different AMH levels could predict the quality of oocytes, the presence of postmaturity and nucleoli $\mathrm{Z}$ score, early cleavage, and ICSI outcomes [7]. Therefore, much information is needed to be explained about the contribution of Antimullerian hormone to oocyte quality, oocyte cytoplasmic maturation and fertilization mechanism.

The aim of this study was to examine hormonal and clinical parameters in women and sperm function parameters in men and to examine the chance of predicting TFF before the day of ICSI in patients with acceptable semen analysis findings in two consecutive fertilization failure cycles.

\section{Material and Methods}

This is a retrospective study with a cross-sectional design at Sisli Memorial IVF Clinic was performed from May 2013 to July 2018 Patient all data were collected from data base of the clinic with the permission of Sisli Memorial Hospital menagement. Because we performed a retrospective analysis of an established clinical procedure in our unit, institutional review board approval was deemed unnecessary. The study population included infertile women 25-37 years old of age who went through serum hormone level tests as well as oocytes retrieval to examine oocyte quantity and quality as a part of IVF procedures. Ovarian stimulation in patients consisted of antagonist protocols were used. Follicular development was monitored and when the maximal diameter of three leading follicles exceeded $18 \mathrm{~mm}, 10000 \mathrm{IU}$ hCG (Pregnyl 5000 IU Amp. Organon) was given intramuscularly. After 36 hours, follicles were aspirated under transvaginal ultrasound guidance with a single lumen aspiration needle. 2 hours post-collection, the oocytes were denuded of their surrounding cumulus cells with hyaluronidase (Vitrolife, Sweden) and mechanical pipeting, which allows a precise determination of nuclear maturation status and oocyte morphology. 91 fertil controls and 32 total fertilization failure in last two cycles, cases with sperm function test results included the study. Routine semen analysis was done by light microscopy according to strict criteria. Morphology was evaluated by Diff Quick staining technique. Serum AMH, E2, Inhibin B and FSH levels were measured on 3th day during the menstrual cycle and HCG day E2 level using ELISA (Beckman/Coulter, USA). Oocyte and zona morphology and maturation level was determined from photographs taken on the day of oocytes retrieval using Nikon eclipse Ti-U software. Dark and irregularities with thick and thin zona pellucida were evaluated as zona anomalies. Patients with no serum AMH data, azoospermia and no mature oocytes retrieved were excluded. Sperm analysis and the sperm function tests are routine procedures in IVF center. The data were analyzed with SPSS 17.0 package program.

\section{Results}

When the data of the groups are examined in the study; total fertilization failure was observed in women and men ages were higher (Table 1). Basal FSH was found to be higher in the FF group and AMH was lower than in fertile controls (Table 1). In the fertilization insufficiency group, more gonadotropin was used for treatment (Table 1). When the oocyte parameters were examined, the average oocyte count and M2 oocyte count were lower in the FF group. In addition, zona pellucida morphology was found to be worse in FF group (Table 2). In Table 3, semen analysis and sperm membrane integrity, DNA fragmentation and sperm maturation analyzes were similar in both groups.

Table 1. Comparison of clinical parameters of fertile control cases and total fertilization failure cases.

\begin{tabular}{llll}
\hline Patients (n) & Fertil controls (91) & Fertilization Failure (TFF) (32) & p \\
\hline Age of women (years) & $30,86 \pm 3,99$ & $33,5 \pm 5,12$ & 0,004 \\
Age of men (years) & $34,88 \pm 4,50$ & $36,93 \pm 5,04$ & 0,041 \\
BMI (kg/m ${ }^{2}$ ) & $25,31 \pm 4,45$ & $26,80 \pm 3,95$ & 0,09 \\
Duration of infertility (years) & $6,88 \pm 4,04$ & $7,96 \pm 4,75$ & 0,224 \\
Basal FSH (IU/ml) & $5,25 \pm 2,05$ & $7,80 \pm 2,05$ & 0,008 \\
Basal LH (IU/ml) & $4,09 \pm 2,16$ & $3,71 \pm 2,47$ & 0,437 \\
Basal E2 (pMol/ml) & $39,27 \pm 18,32$ & $43,28 \pm 14,89$ \\
Inhibin B & $77,31 \pm 59,57$ & $66,21 \pm 46,79$ \\
AMH (ng/ml) & $3,83 \pm 2,86$ & $1,87 \pm 1,74$ & 0,302 \\
Endometrial Thickness (mm) & $10,25 \pm 2,11$ & $8,72 \pm 2,56$ & 0,397 \\
HCG day E2 (pmol/ml) & $1899,5 \pm 1097,26$ & 0,002 \\
Gn (U) & $1971,79 \pm 817,37$ & 0,110 \\
\hline
\end{tabular}

Table 2. The number of mature or immature oocytes, high quality oocytes and zona pellucida abnormalities in fertil or fertilization failure patient groups.

\begin{tabular}{llll}
\hline Patients (n) & Fertil controls (91) & Fertilization failure (32) & P \\
\hline Total oocytes & $9,64 \pm 3,47$ & $4,48 \pm 4,30$ & 0,000 \\
M2 oocytes & $7,63 \pm 3,65$ & $3,19 \pm 3,23$ & 0,000 \\
M1 oocytes & $0,16 \pm 0,52$ & $0,29 \pm 0,73$ & 0,313 \\
GV & $1,06 \pm 1,54$ & $0,51 \pm 0,88$ & 0,063 \\
High quality M2 & $5,08 \pm 3,53$ & $2,77 \pm 3,06$ & 0,008 \\
Normal Zona Pellucida & $7,46 \pm 3,69$ & $2,67 \pm 2,89$ & 0,000 \\
\hline
\end{tabular}


Table 3. Sperm parameters and function tests of the groups.

\begin{tabular}{llll}
\hline & Fertil controls & Fertilization failure & p \\
\hline Concentration (mil/ml) & $51,71 \pm 38,96$ & $65,30 \pm 59,60$ & 0,313 \\
Total motility (\%) & $21,70 \pm 15,72$ & $19,53 \pm 13,61$ & 0,726 \\
Mot A (\%) & $12,32 \pm 12,84$ & $11,15 \pm 11,84$ & 0,766 \\
Mot B (\%) & $6,42 \pm 4,94$ & $4,61 \pm 3,33$ & 0,214 \\
Morphology (\%) & $3,01 \pm 2,79$ & $4,91 \pm 3,84$ & 0,052 \\
HOS (+)(\%) & $62,27 \pm 22,60$ & $76,80 \pm 19,86$ & 0,183 \\
AO test (+) (\%) & $40,61 \pm 18,69$ & $36,25 \pm 16,74$ & 0,668 \\
Chromatin condensation (\%) & $23,80 \pm 19,97$ & $15,66 \pm 10,01$ & 0,502 \\
\hline
\end{tabular}

\section{Discussion}

In this study, it was found that the age of the women was higher, the serum AMH level was below $2 \mathrm{ng} / \mathrm{dl}$, the number of oocytes was less than 5 and the number of M2 oocytes was less than 4 and the number of low zona pellucida normal oocytes in cases with fertilization deficiency.

Because of the many factors involved in the fertilization process, it may be observed that there are patients who have been exposed to complete insemination failure despite the presence of a normal spermatozoon [8-10]. The frequency of total fertilization failure cycles was reported to be $\sim 1-3 \%$ and also characterized by very low oocyte counts [11-13]. In such cases, repeated ICSI treatment has proven to be beneficial however, some patients will have to face repeated fertilization failure despite normal sperm parameters and good ovarian response [3,12, 13].

Successful fertilization is observed in cases with normal oocyte maturation. The proper response of oocyte cytoplasm to sperm PLC $\zeta$ signals is a very important step for fertilization [14]. The ability to produce sequential cytoplasmic calcium releases during fertilization requires several cytoplasmic changes: rearrangement of the endoplasmic reticulum (ER), increase in the number of IP3 receptors, changes in the biochemical properties of receptors (sensitivity to IP3), an increase in the concentration of calcium ions stored in the endoplasmic reticulum (ER) and redistribution binding ER proteins [1519]. Studies on non-fertilized oocytes in IVF / ICSI cycles have shown the presence of abnormal spindles and interphase microtubes indicating that deficiencies in ooplasmic and nuclear components may be a cause of failed fertilization $[20,21]$.

Anti-Müllerian hormone (AMH) is a glycoprotein growth factor secreted by granulosa cells [22]. The normal expression pattern of $\mathrm{AMH}$ is found to be low in the primary follicles, followed by large pre-antral and small antral follicles, followed by a decrease in follicle growth. Based on this expression pattern, it was suggested that the concentration of serum AMH would reflect the number of follicles that grew early as the follicle pool decreased with decreasing growth [23-25]. Previous studies have investigated $\mathrm{AMH}$ gene expression levels in granulosa cells and their correlation with oocyte quality, but the results are inconsistent and contradictory [26-28]. Studies also show that AMH gene expression levels in CCs correlate with AMH concentration in the respective follicular fluid (FF). Anti-mullerian hormone (AMH) is thought to reflect the growth of follicles and the ovarian function [29-31]. In a study conducted by Borges et al. In 2017, it was suggested that serum AMH levels affect the results of ovarian hyperstimulation, it is a useful indicator of oocyte quality and ovarian response to fertilization, but AMH levels do not impair embryo development [32]. In the study of Gupta et al, (2017) it was suggested that antimullerian hormone had an effect on oocyte quality (28). Borges et al suggested that serum levels of AMH are a useful predictor of ovarian response to COS, oocyte quality, and fertilization [32].

Traditionally, the most important measurement in the assessment of ovarian function has been age-independent baseline follicle-stimulating hormone (FSH) levels [31]. Age-specific baseline FSH levels predicted the retrieval of fewer than or equal to four oocytes, with a positive predictive value of $19.5 \%$ and a negative predictive value of $88 \%$ [31]. According the study of Fang and Abdalla $(2015,2004)$ basal FSH levels combined with age (agespecific FSH levels) can be used as a more accurate marker for the ovarian response in women with normal ovarian reserves undergoing IVF-ET, particularly in women $\leq 37$ years old $[30,33]$. In our study, the mean female age in the patient groups was 30.86 in the fertile group and 33.5 in the ff group. In contrast, the mean FSH value was 5.25 and 7.80. According to the number of M2 oocytes, the average oocyte in the ff group was below 4 . In this case, the increase in the basal FSH value in the young patient group has affected the number and quality of oocytes. According to the studies Zona Pellucida dysmorphology is associated with markedly diminished pregnancy and implantation rates in IVF [34]. There is no consensus on the effects of oocyte morphological anomalies on fertilization, embryo quality and pregnancy outcomes [35]. There are also studies showing that abnormal oocytes do not affect fertilization, embryo quality and pregnancy outcomes negatively [36, 37-40].

In conclusion, in the presence of optimal sperm parameters, low M2 oocyte count, age-inappropriate FSH elevation and low $\mathrm{AMH}$ values should be investigated in cases of fertilization failure.

\section{Conflicts of Interest}

Authors declare that there is not any conflict of interest. 


\section{Author Contribution Statement}

\author{
Design of the study: T. I, O. G, S. K. \\ Laboratory documentation: H. Y. \\ Statistics: O. G.
}

\section{Acknowledgements}

This research did not receive any specific grant from any funding agency in the public, commercial or not-for- profit sector. Since our study is a retrospective study, ethical application is not required. However, permission was taken from Şişli Memorial hospital management.

\section{References}

[1] Tesarik, J., Junca, A. M., Hazout, A., Aubriot, F. X., Nathan, C., Cohen-Bacrie, P. and Dumont-Hassan, M. (2000) Embryos with high implantation potential after intracytoplasmic sperm injection can be recognized by a simple, non-invasive examination of pronuclear morphology. Hum. Reprod., 15, 1396-1399.

[2] Ben-Yosef D, Shalgi R. Oocyte activation: lessons from human infertility. Trends Mol Med. 2001 Apr; 7 (4): 163-9. doi: 10.1016/s1471-4914 (01)01957-8. PMID: 11286940.

[3] Tesarik J, Rienzi L, Ubaldi F, Mendoza C and Greco E. Use of a modified intracytoplasmic sperm injection technique to overcome sperm-borne oocyte activation failures. Fertil Steril, 2002, 78, 619-624.

[4] Torra-Massana M, Cornet-Bartolomé D, Barragán M, Durban M, Ferrer-Vaquer A, Zambelli F, Rodriguez A, Oliva R, Vassena R. Novel phospholipase C zeta 1 mutations associated with fertilization failures after ICSI. Hum Reprod. 2019, 1; 34 (8): 1494-1504. doi: 10.1093 /humrep/dez094.

[5] Modarres P, Tavalaee M, Ghaedi K, Nasr-Esfahani MH. An Overview of The Globozoospermia as A Multigenic Identified Syndrome. Int J Fertil Steril. 2019; 12 (4): 273-277. doi: 10.22074/ijfs.2019.5561. Epub 2018 Oct 2.

[6] Zhang Y, Shao L, Xu Y, Cui Y, Liu J, et al. Effect of AntiMullerian Hormone in Culture Medium on Quality of Mouse Oocytes Matured In Vitro. PLoS ONE, 2014, 9 (6): e99393. doi: 10.1371/journal.pone.0099393.

[7] Irez T., Ocal P., Guralp O., Cetin M., Aydogan B., Sahmay S. Different serum anti-Müllerian hormone concentrations are associated with oocyte quality, embryo development parameters and IVF-ICSI outcomes. Arch Gynecol Obstet. 2011; 284 (5): 1295-301. doi: 10.1007/s00404-011-1979-6. Epub 2011 Jul 12.

[8] Tesarik J, Sousa M, Testart J. Human oocyte activation after intracytoplasmic sperm injection. Hum Reprod. 1994 Mar; 9 (3): 511-8. doi: 10.1093/oxfordjournals.humrep.a138537. Erratum in: Hum Reprod 1994 May; 9 (5): 968. PMID: 8006144.

[9] Dozortsev D, Rybouchkin A, De Sutter P, Qian C, Dhont M. Human oocyte activation following intracytoplasmic injection: the role of the sperm cell. Hum Reprod. 1995 Feb; 10 (2): 403-7. doi: 10.1093/oxfordjournals.humrep.a135952. PMID: 7769071.
[10] Ludwig M, Finas DF, al-Hasani S, Diedrich K, Ortmann O. Oocyte quality and treatment outcome in intracytoplasmic sperm injection cycles of polycystic ovarian syndrome patients. Hum Reprod. 1999 Feb; 14 (2): 354-8. doi: 10.1093/humrep/14.2.354. PMID: 10099978.

[11] Jiaen Liu, Zsolt Nagy, Hubert Joris, Herman Tournaye, Paul Devroey, André Van Steirteghem, Andrology: Successful fertilization and establishment of pregnancies after intracytoplasmic sperm injection in patients with globozoospermia, Human Reproduction, Volume 10, Issue 3, March 1995, Pages 626-629, https://doi.org/10.1093/oxfordjournals.humrep.a136000.

[12] Niakan KK, Han J, Pedersen RA, Simon C, Pera RA. Human pre-implantation embryo development. Development. 2012; 139 (5): 829-841. doi: 10.1242/dev.060426.

[13] Seidler, E., Murphy, L., Vaughan, D., \& Sakkas, D. (2019). Fertilization and Complete Fertilization Failure in Human IVF. In G. Kovacs, A. Rutherford, \& D. Gardner (Eds.), How to Prepare the Egg and Embryo to Maximize IVF Success (pp. 224-231). Cambridge: Cambridge University Press. doi: $10.1017 / 9781316756744.019$.

[14] Swain JE, Pool TB. ART failure: oocyte contributions to unsuccessful fertilization. Hum Reprod Update. 2008 SepOct; 14 (5): 431-46. doi: 10.1093/humupd/dmn025. Epub 2008 Jul 5. PMID: 18603645.

[15] Heindryckx B., Elst J. V., Sutter P. D., Dhont M. Treatment option for sperm- or oocyte-related fertilization failure: assisted oocyte activation following diagnostic heterologous ICSI. Human Reproduction, 2005, 20, (8), 2237-2241.

[16] Meerschaut F. V., Nikiforaki D., Heindryckxlow B. Assisted oocyte activation following ICSI fertilization failure. RBMOnline 2014, 28 (5): 560-571.

[17] Neri Q. V., Lee B., Rosenwaks Z., Machaca K., Palermo G. D. Understanding fertilization through intracytoplasmic sperm injection (ICSI) Cell Calcium, 2014, 55 (1), 24-37.

[18] Vanderheyden V., Wakaib T. Bultynck G., De Smedt H.,. Parysa J. B., Fissore R. A. Regulation of inositol 1,4,5trisphosphate receptor type 1 function during oocyte maturation by MPM-2 phosphorylation. Cell Calcium Volume 46, Issue 1, July 2009, Pages 56-64.

[19] Goud P, Goud A, Van Oostveldt P, Van der Elst J, Dhont M. Fertilization abnormalities and pronucleus size asynchrony after intracytoplasmic sperm injection are related to oocyte postmaturity. Fertil Steril. 1999 Aug; 72 (2): 245-52. doi: 10.1016/s0015-0282 (99)00231-9. PMID: 10438989.

[20] Combelles CM, Morozumi K, Yanagimachi R, Zhu L, Fox JH, Racowsky C. Diagnosing cellular defects in an unexplained case of total fertilization failure. Hum Reprod. $2010 \mathrm{Jul} ; 25$ (7): 1666-71. doi: 10.1093/humrep/deq064. Epub 2010 May 15. PMID: 20472911.

[21] Kovacic B, Vlaisavljević V. Influence of atmospheric versus reduced oxygen concentration on development of uman blastocysts in vitro: a prospective study on sibling oocytes. Reprod Biomed Online. 2008 Aug; 17 (2): 229-36. doi: 10.1016/s1472-6483 (10)60199-x. PMID: 18681997.

[22] Shahrokhi SZ, Kazerouni F, Ghaffari F. Anti-Müllerian Hormone: genetic and environmental effects. Clin Chim Acta 2018; 476: 123-129. 
[23] Lie Fong S, Visser JA, Welt CK, et al. Serum anti-Müllerian hormone levels in healthy females: a nomogram ranging from infancy to adulthood. J Clin Endocrinol Metab 2012; 97: $4650-4655$.

[24] Tolikas A, Tsakos E, Gerou S, et al. Anti-Müllerian Hormone (AMH) levels in serum and follicular fluid as predictors of ovarian response in stimulated (IVF and ICSI) cycles. Hum Fertil 2011; 14 (4): 246-253.

[25] Borges E., Braga D. P. A. F., Setti A., Figueira R. C., and Iaconelli $\mathrm{A}$. The predictive value of serum concentrations of anti-Müllerian hormone for oocyte quality, fertilization, and implantation. JBRA Assist Reprod. 2017; 21 (3): 176-182. doi: 10.5935/1518-0557.20170035 PMCID: PMC5574637 PMID: 28837024.

[26] Catteau-Jonard S, Jamin SP, Leclerc A, et al. Anti-Müllerian hormone, its receptor, FSH receptor, and androgen receptor genes are overexpressed by granulosa cells from stimulated follicles in women with polycystic ovary syndrome. J Clin Endocrinol Metab 2008; 93 (11): 4456-4461.

[27] Kedem-Dickman A, Maman E, Yung Y, et al. Anti-Müllerian hormone is highly expressed and secreted from cumulus granulosa cells of stimulated preovulatory immature and atretic oocytes. Reprod Biomed Online 2012; 24: 540-546.

[28] Gupta S., Karuputhula N. B., Kumar N., Srivastava A., Singh B., Dubey K. Correlation of basal serum anti-Mullerian hormone level with oocyte quality and embryo development potential in women undergoing IVF-ICSI. Fertility Science and Research, 2017, 4, 2, 112-116.

[29] Velásquez A., Mellisho E., Ovidio F. C., Rodríguez-Álvarez L. Effect of BMP15 and/or AMH during in vitro maturation of oocytes from involuntarily culled dairy cows. Molecular Reproduction and Development, 2019, 86, 2, 209-223, https://doi.org/10.1002/mrd.23096

[30] Fang T., Su Z., Wang L., Yuan P., Li R., Ouyang N., Zheng L., and Wang W. Predictive value of age-specific FSH levels for IVF-ET outcome in women with normal ovarian function Reprod Biol Endocrinol. 2015; 13: 63. Published online 2015 Jun 17. doi: 10.1186/s12958-015-0056-6 PMCID: PMC4470037 PMID: 26082101 DOI 10.1186/s12958-0150056-6.
[31] Barad DH, Weghofer A, Gleicher N Age-specific levels for basal follicle-stimulating hormone assessment of ovarian function. Obstet Gynecol. 2007; 109 (6): 1404-10.

[32] Borges E, Braga DPAF, Setti A, Figueira RC, Iaconelli A Jr. The predictive value of serum concentrations of antiMüllerian hormone for oocyte quality, fertilization, and implantation. JBRA Assist Reprod. 2017; 21 (3): 176-182. Published 2017 Sep 1. doi: 10.5935/1518-0557.20170035.

[33] Abdalla H, Thum MY. An elevated basal FSH reflects a quantitative rather than qualitative decline of the ovarian reserve. Hum Reprod. 2004 Apr; 19 (4): 893-8. doi: 10.1093/humrep/deh141. Epub 2004 Mar 11. PMID: 15016786.

[34] Sauerbrun-Cutler MT, Vega M., Breborowicz A., Gonzales E., Stein D., Lederman M., and Keltz M. Oocyte zona pellucida dysmorphology is associated with diminished in-vitro fertilization success. J Ovarian Res. 2015; 8: 5. Published online 2015 Feb 27. doi: 10.1186/s13048-014-0111-5 PMCID: PMC4355133 PMID: 25823613.

[35] Xu H. Deng K. Luo Q. Chen J. Zhang X. Wang X. Diao H. ZhangC. High Serum FSH is Associated with Brown Oocyte Formation and a Lower Pregnacy Rate in Human IVF Parctice Cellular Physiology and Biochemistry 2016; 39: 677-684 https://doi.org/10.1159/000445658

[36] Rienzi L, Vajta G, Ubaldi F: Predictive value of oocyte morphology in human IVF: a systematic review of the literature. Hum Reprod Update 2011; 17: 34-45.

[37] Alikani M, Palermo G, Adler A, Bertoli M, Blake M, Cohen J: Intracytoplasmic sperm injection in dysmorphic human oocytes. Zygote 1995; 3: 283-288.

[38] Ten J, Mendiola J, Vioque J, de Juan J, Bernabeu R: Donor oocyte dysmorphisms and their influence on fertilization and embryo quality. Reprod Biomed Online 2007; 14: 40-48.

[39] Rienzi L, Balaban B, Ebner T, Mandelbaum J: The oocyte. Hum Reprod 2012; 27: 12-21.

[40] Van Blerkom J, Henry G: Oocyte dysmorphism and aneuploidy in meiotically mature human oocytes after ovarian stimulation. Hum Reprod 1992; 7: 379-390. 